\title{
Characterization of the Streptococcus gordonii chromosomal region immediately downstream of the glucosyltransferase gene
}

\author{
M. M. Vickerman, ${ }^{1}$ P. E. Minick ${ }^{2}$ and N. M. Mather ${ }^{2}$ \\ Author for correspondence: M. M. Vickerman. Tel: +1 317278 3250. Fax: +1 3172786244 \\ e-mail:mvickerm@iupui.edu
}

Department of Oral Surgery and Hospital Dentistry, School of Dentistry 1,2 and Department of Microbiology and Immunology, ${ }^{1}$ School of Medicine, Indiana University, Indianapolis, IN 46202, USA
The Streptococcus gordonii glucosyltransferase gene, gtfG, is positively regulated by the upstream determinant rgg. In the present study, two ORFs, transcribed on the opposite DNA strand, were identified immediately downstream of gtfG. The first, designated dsg, shares a convergent putative transcriptional terminator with gtfG, and encodes a predicted 46 kDa transmembrane protein similar to the Yersinia enterocolitica TrsA involved in polysaccharide biosynthesis. Insertional inactivation of dsg resulted in only $\sim 60 \%$ of the parental level of glucosyltransferase activity. The 870 bp gene 5' to $d s g$ is similar to the gtfG regulatory determinant. Designated rggD, this rgglike determinant downstream of gtfG encodes a putative $33.6 \mathrm{kDa}$ cytoplasmic protein. Despite their sequence similarity, the functions of $r g g$ and $r g g D$ appear specific. Strains in which rggD was insertionally inactivated and strains containing plasmid-borne rggD had parental levels of glucosyltransferase activity. Northern blot hybridization analyses showed $\sim 1.3 \mathrm{~kb}$ dsg-specific and $\sim 1.0 \mathrm{~kb}$ rggD-specific mRNA transcripts associated with this region; no polycistronic transcript was observed. Although rgg-like gene products have been demonstrated to function as positive transcriptional regulators of adjacent genes in several streptococcal species, Northern blot analysis suggested that $r g g D$ did not influence the transcription of $d s g$ or the divergent downstream ylbN-like determinant under the conditions in the present study. Comparison of this $\mathbf{S}$. gordonii chromosome region to other streptococcal genomes, which do not contain the rgg/rggD-flanked region involved in glucan synthesis, raised intriguing possibilities about the origins of this chromosomal region, and also suggested that rggD might regulate a distally located gene.

Keywords: oral streptococci, glucans, $r g g$

\section{INTRODUCTION}

Glucan polymers synthesized by the extracellular glucosyltransferase (GTF) enzymes of oral streptococci are associated with the ability of bacteria to accumulate on tooth surfaces in dental plaque. Mutans streptococci and Streptococcus salivarius have multiple enzymes in each strain that synthesize primarily water-soluble or water-insoluble glucans from sucrose. Although en-

Abbreviations: GTF, glucosyltransferase; IPCR, inverse PCR; Spp, sucrosepromoted phenotype.

The nucleotide sequence of the $S$. gordonii chromosomal region reported in this paper has been appended to the gtfG entry in GenBank with accession number U12643. vironmental factors have been shown to influence expression of gtf genes in these species, no genetic regulatory determinants for their gtf genes have been identified. In contrast, the commensal species Streptococcus gordonii has a single GTF enzyme that synthesizes both $\alpha 1,3$ - and $\alpha 1,6$-linked glucans (Grahame \& Mayer, 1984; Haisman \& Jenkinson, 1991). Moreover, $S$. gordonii has a positive regulatory determinant, rgg, located immediately upstream from the GTF structural gene, gtfG, which encodes a trans-acting product that increases $g t f G$ transcription (Sulavik et al., 1992). Both rgg and gtfG have their own putative promoter and ribosome-binding site. Three mRNA transcripts have been associated with the $\mathrm{rgg} / \mathrm{gtfG}$ locus: a $1.1 \mathrm{~kb}$ rgg-specific transcript, a $5.5 \mathrm{~kb} g t f G$ - 
specific transcript and a $6.5 \mathrm{~kb} \mathrm{rgg} / \mathrm{gtfG}$ polycistronic transcript (Sulavik \& Clewell, 1996).

At the time rgg was first identified, there were no similar genes in the genetic databases (Sulavik et al., 1992). Since then, determinants with significant similarity to $r g g$ have been identified in a number of related bacterial species. Nucleotide sequence and Southern blot hybridization analyses have identified $r g g$-like determinants in Streptococcus oralis (Fujiwara et al., 2000) and Streptococcus sanguis (Vickerman et al., 1995), early colonizers of dental plaque (Frandsen et al., 1991) which, like S. gordonii, appear to have only one GTF enzyme; these $r g g$-like determinants appear to be located near their gtf determinants, suggesting that GTF expression in these species may be regulated in a manner similar to that of S. gordonii (Vickerman et al., 1995). rgg-like determinants have also been characterized in other streptococcal (Lyon et al., 1998; Chaussee et al., 1999; Qi et al., 1999) and lactococcal (Sanders et al., 1998) species, and have been found to regulate a variety of proteins with different functions. These data suggest that $r g g$-like genes are members of a family of important streptococcal regulatory determinants.

Genetic data suggest that the regulation of $S$. gordonii GTF activity is complex and may involve genes in addition to $r g g$ and $g t f G$. Both $r g g$ and $g t f G$ are preceded by DNA inverted repeats, suggestive of possible regulatory factor binding sites (Sulavik et al., 1992). Two distinct genetic loci that influence GTF activity have been identified by chemical mutagenesis (Haisman \& Jenkinson, 1991). Nucleotide sequence analysis has shown that $S$. gordonii strains with only $20-30 \%$ of the parental level of GTF activity have no differences in the $5.95 \mathrm{~kb}$ genome region encoding rgg, gtfG and their immediate flanking regions (Vickerman et al., 1997a). Because functionally linked genes are often located in close proximity on bacterial chromosomes, it was hypothesized that genes flanking the $\mathrm{rgg} / \mathrm{gtfG}$ locus could be involved in GTF regulation. However, insertional inactivation of the $\operatorname{lem} A$ and $h t p X$ genes immediately upstream of $\mathrm{rgg}$ did not affect the level of GTF expression (Vickerman et al., 2001). Therefore, the present study was undertaken to examine the $S$. gordonii chromosomal region downstream of $g t f G$ and to determine potential influences of any genes identified in this region on GTF activity.

\section{METHODS}

Bacteria, medium, and culture conditions. Bacterial strains and plasmids used in this study are shown in Table 1. All strains were stored at $-70^{\circ} \mathrm{C}$ in $50 \%$ (v/v) glycerol. $S$. gordonii strains were grown in Todd-Hewitt broth (TH; Difco) or in a defined FMC (Terleckyj et al., 1975) medium and incubated at $36^{\circ} \mathrm{C}$ in an anaerobic chamber (Coy Industries) with a gas mixture of $85 \% \mathrm{~N}_{2}, 10 \% \mathrm{H}_{2}, 5 \% \mathrm{CO}_{2}$. The sucrose-promoted colony phenotype $\left(\mathrm{Spp}^{+}\right.$for parental strain Challis CH1) was determined on $3 \%$ (w/v) sucrose TH agar plates incubated for $48 \mathrm{~h}$ in $5 \% \mathrm{CO}_{2}$. This is a hard colony phenotype which has been associated with glucan synthesis (Tardif et al., 1989). S. gordonii strains carrying replicative or chromosomally integrated plasmids were selected and grown with $5 \mu \mathrm{g}$ erythromycin $\mathrm{ml}^{-1}$. Escherichia coli DH5 $\alpha$ (Invitrogen Life Technologies) strains used to construct pVA891 derivatives for streptococcal integration were grown in Luria-Bertani medium containing $300 \mu \mathrm{g}$ erythromycin $\mathrm{ml}^{-1}$ and incubated aerobically at $37^{\circ} \mathrm{C}$. Growth phase was determined by measuring $\mathrm{OD}_{520}$ or $\mathrm{OD}_{600}$ for FMC and TH broth cultures, respectively, in a Pharmacia Ultraspec 2000 spectrophotometer.

DNA isolation and manipulations. DNA preparation and manipulations were done according to standard molecular biology procedures (Ausubel et al., 1987). Modifications for $S$. gordonii included growing strains in $0.5 \%(\mathrm{w} / \mathrm{v})$ glycine and incubating cells with mutanolysin and lysozyme to facilitate lysis, as described by Sulavik et al. (1992). Plasmid DNA was prepared using Qiagen purification columns according to the manufacturer's directions. Double-stranded PCR products were obtained using Elongase enzyme (Invitrogen Life Technologies). In some cases, oligonucleotide primers were designed with engineered restriction sites (indicated by underlining: see Results) and random flanking 5' nucleotides (shown in lower-case letters) to ensure efficient digestion. DNA fragments were eluted from agarose gels with a QiaexII bead kit (Qiagen). For cloning, DNA fragments were digested with appropriate restriction enzymes, ligated with T4 DNA ligase into convenient restriction sites of the vector and transformed into either $\mathrm{CaCl}_{2}$-competent E. coli $\mathrm{DH} 5 \alpha$ or S. gordonii cells made competent (Lawson \& Gooder, 1970) with horse serum.

Southern blot hybridization analyses. S. gordonii chromosomal DNA was digested with appropriate restriction enzymes, electrophoresed on $0.7 \%$ agarose gels and transferred to a Hybond-N membrane via capillary action under neutral conditions (Ausubel et al., 1987). Probe DNA was labelled with digoxigenin-dUTP, hybridized to membranes and washed under stringent conditions. Hybridized probe was detected by chemiluminescence with the Genius System (Roche Molecular Biochemicals), according to the manufacturer's directions.

Recovery of S. gordonii chromosomal DNA downstream of gtfG. Nucleotide sequencing of the downstream region was originally done using the E. coli plasmid pAMS21, which carries the downstream $3.4 \mathrm{~kb}$ HindIII fragment of pAMS40 subcloned in pBluescript (Vickerman et al., 1997b; Fig. 1a). However, difficulty identifying ORFs raised the possibility that there were difficulties cloning this region. Therefore, using primers derived from the pAMS21 sequence, three independent identical PCR products from this region were sequenced directly. The results indicated that deletions had occurred in the ORF immediately downstream of $g t f G$ in pAMS21. Subsequent attempts to clone these PCR products confirmed that deletions occurred in E. coli cloning vectors. Consequently, the nucleotide sequence of the region downstream of $g t f G$ was determined directly from PCR products. At least three overlapping independent products were sequenced for each region.

Inverse PCR (IPCR) was used to recover additional downstream DNA. A Southern blot of the S. gordonii chromosome showed that the $\sim 600 \mathrm{bp} \mathrm{SstI-HindIII} \mathrm{fragment,} \mathrm{located} \mathrm{at}$ the $3^{\prime}$ end of pAMS40 (Fig. 1a), hybridized to a $\sim 3 \mathrm{~kb} \mathrm{BspHI}$ chromosomal fragment. Therefore, chromosomal DNA was digested with $B s p \mathrm{HI}$, electrophoresed on $0.7 \%$ agarose and fragments ranging from 3 to $4 \mathrm{~kb}$ were eluted, re-circularized and used as a template for IPCR. Amplification with primers 
Table 1. Bacterial strains and plasmids

\begin{tabular}{|c|c|c|}
\hline Strain/plasmid & Relevant characteristics & Reference \\
\hline \multicolumn{3}{|l|}{ Bacteria } \\
\hline $\mathrm{CH} 1$ & S. gordonii parental strain Challis $\mathrm{CH} 1$ & Tardif et al. (1989) \\
\hline $\mathrm{CH} 8942$ & $\begin{array}{l}\text { CH1 with insertional inactivation of } d s g \text { via chromosomally } \\
\text { integrated pMI8942; } \mathrm{Erm}^{\mathrm{R}}\end{array}$ & This study \\
\hline CH8961 & $\begin{array}{l}\text { CH1 with insertional inactivation of } r g g D \text { via chromosomally } \\
\text { integrated pMI8961; } \text { Erm }^{\mathrm{R}}\end{array}$ & This study \\
\hline DS512 & $\begin{array}{l}\mathrm{CH} 1 \text { with premature translational stop in } r g g \text {, with } \sim 3 \% \text { of the } \\
\text { parental level of GTF activity }\end{array}$ & Sulavik et al. (1992) \\
\hline \multicolumn{3}{|l|}{ Plasmids } \\
\hline pVA749 & Streptococcal cloning vector; $\mathrm{Em}^{\mathrm{R}}$ & Macrina et al. (1981) \\
\hline pAMS40 & $9 \cdot 2 \mathrm{~kb}$ region $\mathrm{rgg} / \mathrm{gtfG}$ in HindIII site of pVA749 & Vickerman et al. (1995) \\
\hline pAMS57 & rgg cloned into HaeIII site of pVA749 & Sulavik et al. (1992) \\
\hline pMI226 & rggD cloned into HaeIII site of pVA749 & This study \\
\hline pVA891 & E. coli vector, no streptococcal origin of replication, $\mathrm{Em}^{\mathrm{R}}$ & Macrina et al. (1983) \\
\hline pMI8942 & pVA891 with 315 bp internal fragment of $d s g$ & This study \\
\hline pMI8961 & pVA891 with 322 bp internal fragment of $r g g D$ & This study \\
\hline pAMS21 & $3.4 \mathrm{~kb}$ HindIII fragment of pAMS40 cloned into pBluescript $\mathrm{SK}^{+} ; \mathrm{Ap}^{\mathrm{R}}$ & Vickerman et al. (1997b) \\
\hline
\end{tabular}

5'-CTCTCACGAATCTTCAACAC-3' and 5'-GAAAAGCAGCTTCGAGAGC-3' generated a $\sim 2.8 \mathrm{~kb}$ product (Fig. 1a) which was sequenced directly.

Nucleotide sequence determination and analysis. Both strands of the DNA template were sequenced using a PRISMReady Reaction Dye Deoxy Terminator Sequencing kit (Applied Biosystems) and an automated DNA sequencer (model 373, Applied Biosystems), using customized oligonucleotide primers. Searches for homologues to $S$. gordonii nucleotide and amino acid sequences were done using the BLAST algorithm (Altschul et al., 1990) on the National Center for Biotechnology Information database (http:// www.ncbi.nlm.nih.gov), streptococcal genome databases at the University of Oklahoma (http://genome.ou.edu) and The Institute for Genomic Research database (http:// www.tigr.org). Comparisons of entire genes and their encoded proteins were done using MacVector (version 7.0, Oxford Molecular Group) and the GCG package (Wisconsin Package version 10) for nucleotide and protein similarity. Encoded signal peptide and transmembrane helices were predicted with the Signal P (Nielsen et al., 1997) and TmPred (Hofmann \& Stoffel, 1993) programs. Potential protein structural motifs were identified via the pfam database (Bateman et al., 2000).

Insertional inactivation of ORFs. Oligonucleotide primers with engineered flanking restriction sites and translational stops were used with pAMS40 template to produce internal gene fragments by PCR. The resulting fragments were digested and directionally cloned into the BamHI and HindIII sites of pVA891 (Macrina et al., 1983). After construction and verification in E. coli $\mathrm{DH} 5 \alpha$, purified plasmid DNA was transformed into strain $\mathrm{CH} 1$. Putative $S$. gordonii transformants with the expected plasmid insertion were verified by two methods. Southern blots of digested chromosomal DNA were probed with both pVA891 and the cloned internal gene fragment. Transformant strains that had the expected hybridization patterns were confirmed by direct sequence analysis of PCR products. Primers designed to anneal adjacent to the BamHI and HindIII sites of the vector $\left(5^{\prime}\right.$-ACGATGCGTCCGGCGTAGAG-3' and 5'-AGGTGCTGACTTTCAACTGC-3', respectively) were used with primers designed to anneal to $S$. gordonii chromosomal sequences flanking the region of each expected plasmid insertion. Resulting PCR products with each transformant template were sequenced to confirm the correct integration of each plasmid for gene disruption.

Determination of GTF activity. Relative amounts of GTF activity for each strain were measured via glucan production in acrylamide gels, as described by Tardif et al. (1989). Briefly, strains to be compared were grown to the same mid- to lateexponential stage $\left(\mathrm{OD}_{520} \sim 1 \cdot 6\right)$. Cell pellets were extracted with $1 \%$ (w/v) SDS (Vickerman \& Clewell, 1997) and equal volumes of cell extracts and cell-free culture supernatants were run on an $8.75 \%$ acrylamide SDS-PAGE. After electrophoresis, gels were incubated overnight at $37^{\circ} \mathrm{C}$ in a solution of $3 \%$ sucrose and $0.5 \%$ Triton X-100 in $10 \mathrm{mM}$ sodium phosphate ( $\mathrm{pH} \mathrm{6.8).} \mathrm{The} \mathrm{resulting} \mathrm{glucan} \mathrm{bands} \mathrm{were} \mathrm{stained}$ with pararosaniline, as described by Tardif et al. (1989). Band intensities reflect the relative amount of GTF protein and activity (Vickerman et al., 1996). Relative GTF activity for each strain was determined via laser densitometry (LKB Ultrascan XL) in at least four independent gels. GTF activity was determined as a percentage of the parental strain activity (set at $100 \%$ for the parental strain on each gel).

Northern hybridization analyses. $S$. gordonii cells were grown in $0.5 \%(\mathrm{w} / \mathrm{v})$ glycine medium to the same density. Total RNA was prepared using a Purescript RNA isolation kit (Gentra Systems) essentially to the manufacturer's directions, but with the following modifications: Cell pellets were resuspended in $16.1 \mathrm{mg}$ lysozyme $\mathrm{ml}^{-1}$ and 100 units of mutanolysin in GET buffer $(50 \mathrm{mM}$ glucose, $10 \mathrm{mM}$ EDTA, $25 \mathrm{mM}$ Tris $/ \mathrm{HCl}, \mathrm{pH} \mathrm{8.0)}$ and incubated at $37^{\circ} \mathrm{C}$ for $10 \mathrm{~min}$. After addition of the SDS solution, cells were incubated on ice for $5 \mathrm{~min}$, followed by $30 \mathrm{~s}$ at $65^{\circ} \mathrm{C}$ for lysis. Prepared RNA was further purified with two phenol/chloroform/isoamyl alcohol extractions and an ethanol precipitation. Equal amounts of RNA were electrophoresed on a $2 \%$ formaldehyde $/ 1 \%$ agarose gel, transferred to Hybond-N membranes, probed with digoxigenin-labelled DNA fragments and washed under stringent conditions, according to the instructions for the Genius system (Roche Biochemicals). 
All solutions were prepared using sterilized dimethyl pyrocarbonate-treated water.

\section{RESULTS}

\section{Identification and preliminary characterization of $d s g$}

Immediately downstream of $g t f G$ is a $1236 \mathrm{bp}$ ORF on the opposite reading strand that shares the gtfG putative transcriptional terminator (Fig. 1a). This determinant, designated $d s g$, encodes a putative $46 \mathrm{kDa}$ transmembrane protein. Although $d s g$ does not have highly significant similarity to genes of known function in genomic databases, $d s g$ is $51 \%$ similar to a Lactococcus lactis plasmid-borne gene encoding a hypothetical protein of unknown function. Although no other highprobability homologues were found in GenBank, the encoded Dsg protein is $44 \%$ similar and shares structural similarities with the protein encoded by the $\operatorname{trs} A$ gene of Yersinia enterocolitica (Fig. 2; Skurnik et al., 1995). TrsA is a $47 \mathrm{kDa}$ lipopolysaccharide O-side chain transferase involved in LPS biosynthesis and transport in the Gram-negative cell wall. The trsA gene is a member of the $w z x$ (formerly $r f b X$; Reeves et al., 1996) family of polysaccharide biosynthetic genes. Difficulties in cloning members of this gene family due to deletions, as were seen with $d s g$, have been noted (Schnaitman \& Klena, 1993). The sequence similarity suggests that $d s g$ may serve a related function involving extracellular polysaccharides and transport in the Gram-positive $S$. gordonii.

To examine potential functions of $d s g$, S. gordonii strains were constructed in which $d s g$ was insertionally

(a)

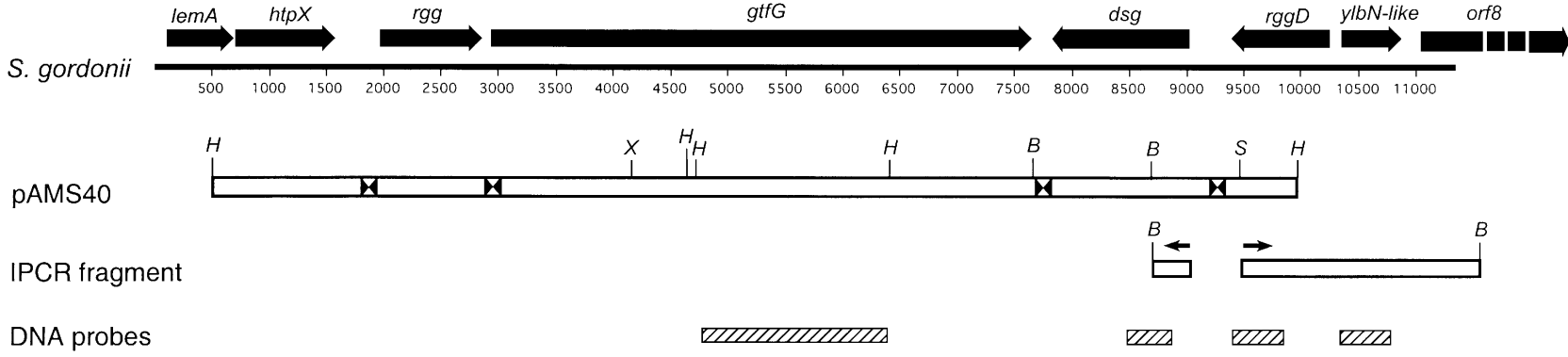

(b)
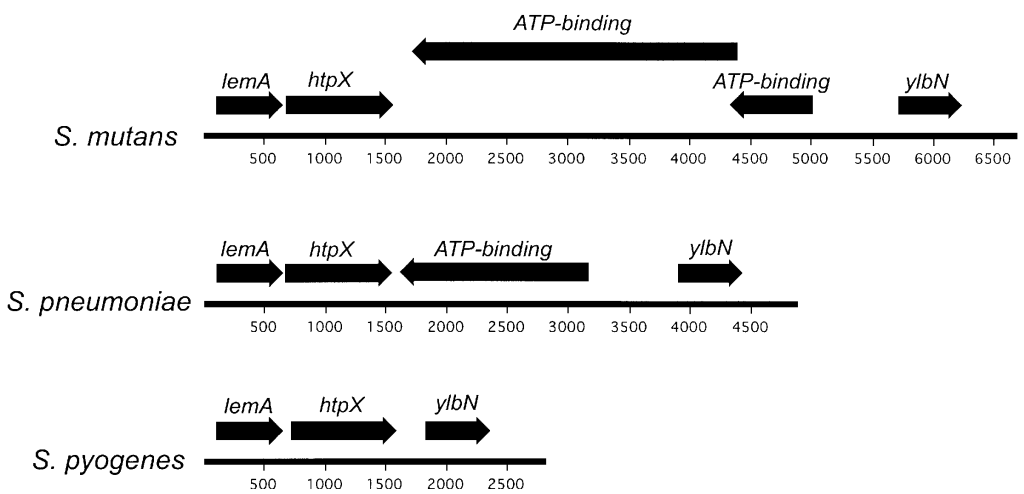

Fig. 1. (a) Schematic diagram depicting the $S$. gordonii chromosomal regions flanking rgg and gtfG, including the upstream lemA and $h t p X$ genes (Vickerman et al., 2002; GenBank accession number AF017421), the $9 \cdot 2 \mathrm{~kb}$ region cloned in pAMS40 (Vickerman et al., 1995) and the IPCR fragment used in the present studies to determine the nucleotide sequence downstream of gtfG. The large arrows above the restriction digest maps indicate the position and direction of the DNA reading strand for each ORF. Relevant restriction sites are represented by $\mathrm{H}($ HindIII), X $(X b a l), B(B s p H I)$ and S (Sstl). Regions of dyad symmetry, which may play functional roles, are designated by inverted arrows within the pAMS40 map. The position and direction of oligonucleotide primers used for IPCR are indicated by small arrows above the mapped product; positions and sizes of DNA probes used for hybridization analyses are indicated by diagonal lines. (b) Schematic diagrams of streptococcal genomic regions containing the $h t p X$ - and $y / b N$-like genes. Reading directions and gene sizes are depicted by large arrows above the base pair numbers. S. pyogenes data are from Feretti et al. (2001); S. pneumoniae data are from The Institute of Genomic Research (http://www.tigr.org); S. mutans data are from the unfinished S. mutans genome site at the University of Oklahoma (http://www.genome.ou.edu/smutans.html). Gene designations lemA, $h t p X$ and $y l b N$ are based on sequence similarity in genome annotations. S. mutans and S. pneumoniae each have genes encoding putative $A B C$ transporter ATP-binding proteins in the opposite reading strand between $h t p X$ - and $y l b N$-like genes. 


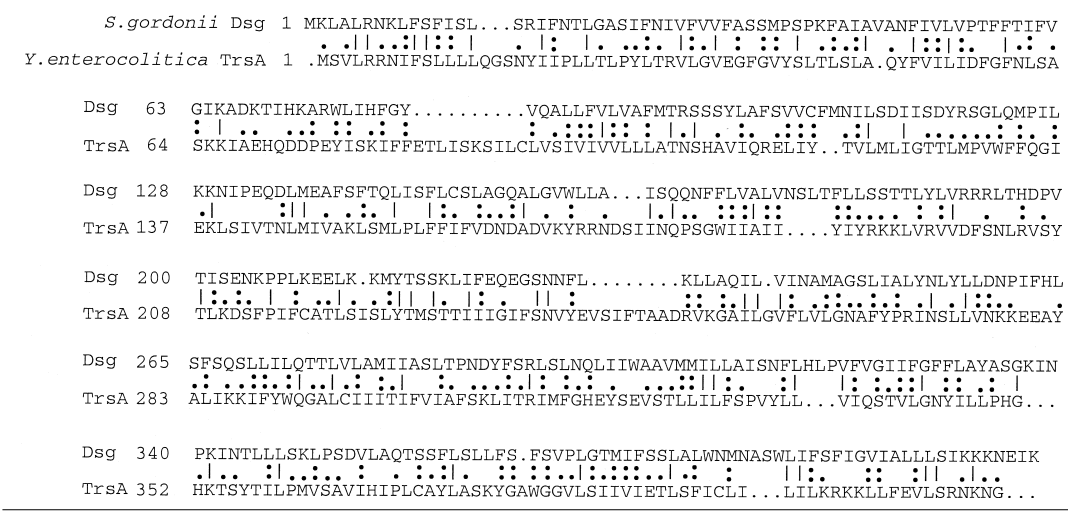

Fig. 2. Comparison of the amino acids of the encoded S. gordonii Dsg protein with the $Y$. enterocolitica $47 \mathrm{kDa}$ TrsA protein, using the GCG gap program with the Blosum62 scoring matrix. The proteins are $44.25 \%$ similar. Identical amino acids are designated with a vertical line. Similar amino acids with a scoring matrix greater than 2 are represented by a double dot; those with a value of 1 are represented by a single dot. Computer analysis programs predict that both proteins have similar signal sequences and 11 transmembrane regions with a $\mathrm{NH}_{2}$-terminus outside orientation. Predicted pl values are 9.77 and 9.52 for Dsg and TrsA, respectively. (a)

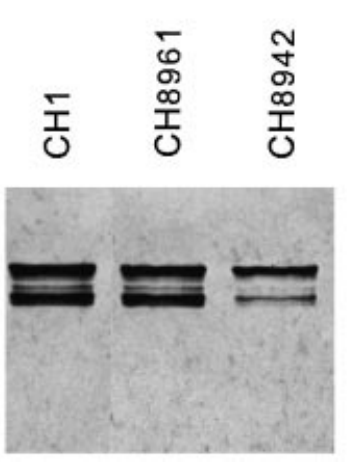

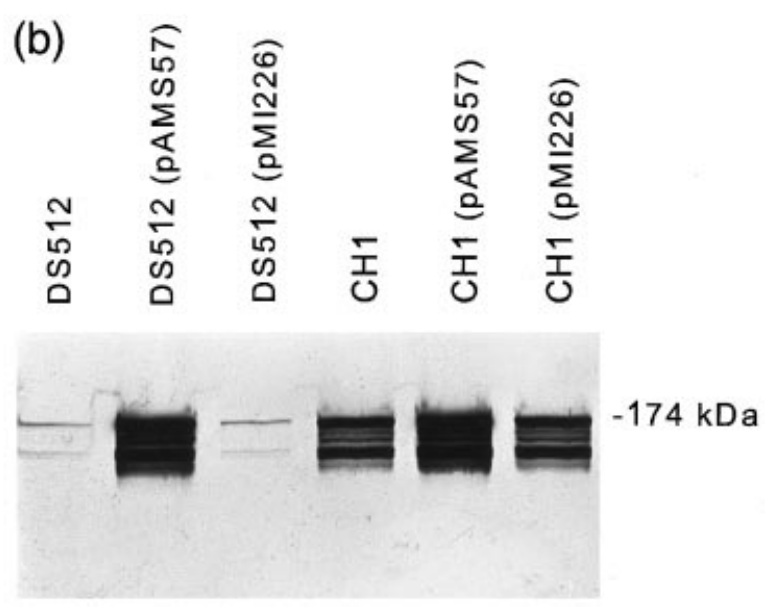

Fig. 3. GTF activities of $S$. gordonii strains. Bacteria were grown in FMC medium to the same cell density and equal volumes of culture supernatants were run on an $8.75 \%$ acrylamide SDS-PAGE. Gels were incubated with sucrose and Triton X-100 detergent at $37^{\circ} \mathrm{C}$. Synthesized glucan bands were treated with periodic acid and stained with pararosaniline. Extracellular GTF from culture supernatants is shown in each gel. Results for cell-associated GTF activity, which made up less than $10 \%$ of the total GTF activity for each strain, were proportional to the extracellular activity for each strain (data not shown). The position of the $174 \mathrm{kDa}$ native protein band is indicated. The lower molecular mass forms of GTF, with various levels of GTF activity, are thought to be due to the degradation of the native enzyme by endogenous proteases and occur even in the presence of protease inhibitors, as described by Grahame \& Mayer (1984) and Vickerman \& Clewell (1997). Strain names are shown above each lane. Gels shown are representative of a minimum of four independent experiments. (a) Effects of insertional inactivation of $d s g$ and rggD. Comparison of strains $\mathrm{CH} 1$ and CH8961 indicates that insertional inactivation of rggD did not affect the level of GTF activity, whereas inactivation of dsg in strain $\mathrm{CH} 8942$ resulted in a decrease to about $60 \%$ of the parental level. (b) Effects of plasmid-borne rgg and rggD. Although rgg in pAMS57 increased the GTF activity of strain CH1 and the rgg-mutant strain DS512, rggD in pMI226 did not affect the level of GTF activity in either strain.

inactivated. Oligonucleotide primers 5'-taGGATCCTTAGGAGTTTAGGCTCTTGGCA-3' and 5'-tag AAGCTTGGGGTTGTCTAGCTAATAGAG-3' were used to amplify an internal region of $d s g$ by PCR. For each primer, underlined bases indicate engineered restriction sites; lower-case letters indicate random flanking $5^{\prime}$ nucleotides; the bold $\mathrm{T}$ in the second primer was added to create a translational stop site in the integrated plasmid to avoid possible read-through from vector sequences. The $315 \mathrm{bp}$ product was cloned into pVA891 and the resulting plasmid, pMI8942, was transformed into strain $\mathrm{CH} 1$. The representative confirmed transformant strain $\mathrm{CH} 8942$, in which $d s g$ was insertionally inactivated, was further characterized.

\section{Disruption of $d s g$ influences GTF activity}

Colonies of strain CH8942 had the hard, cohesive Spp ${ }^{+}$ colony phenotype on sucrose agar plates, indicating that they synthesized glucan polymers. However, the colonies were macroscopically more glassy and translucent than parental strain $\mathrm{CH} 1 \mathrm{Spp}^{+}$colonies, suggesting some difference in the GTF activities of these two strains. Scanning of GTF activity gels indicated that strain $\mathrm{CH} 8942$ only had $57 \cdot 4 \pm 8 \cdot 2 \%$ SD of the extracellular GTF activity of strain CH1 (Fig. 3a). Similar results were seen for cell-associated GTF activity: strain CH8942 had only $53 \cdot 0 \pm 8 \cdot 4 \%$ SD the parental level of cell-associated GTF activity. However, Northern blots 
(a)

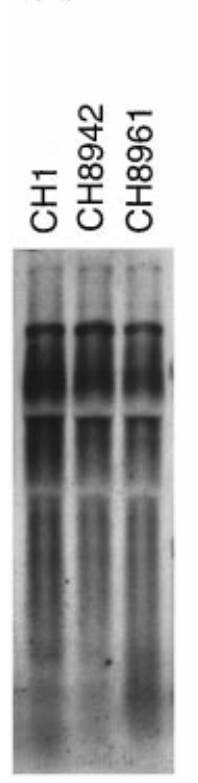

(b)
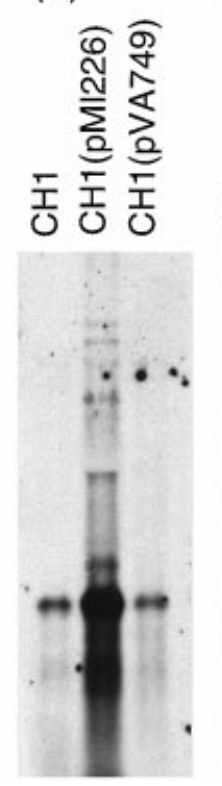

(c)

(d)
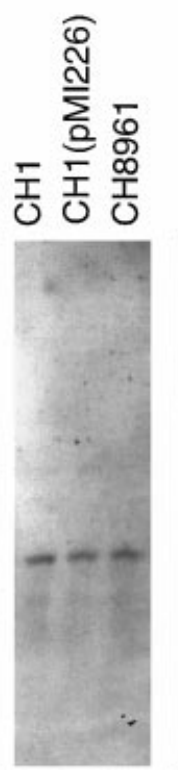
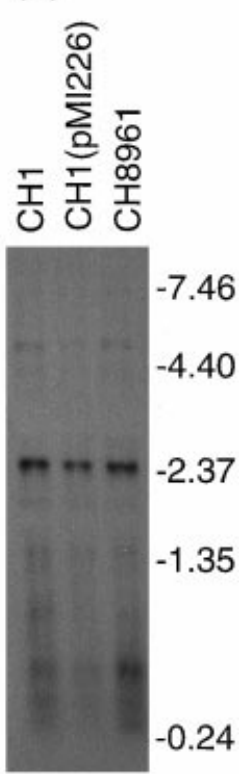

Fig. 4. Identification of $S$. gordonii transcripts by Northern blot analysis. Equal amounts of total RNA from S. gordonii strains (listed above each lane) were electrophoresed on $2 \%$ formaldehyde- $1 \%$ agarose gels, transferred to nylon membranes and hybridized with digoxigenin-labelled probes for internal regions of $g t f G, r g g D, d s g$ and $y / b N$-like genes (represented by hatched bars in Fig. 1a). (a) gtfG-probed RNA of strains $\mathrm{CH} 1, \mathrm{CH} 8942$ and $\mathrm{CH} 8961$ indicates that the levels of the $5.4 \mathrm{~kb}$ gtfG-specific and the $6.4 \mathrm{~kb}$ rgg/gtfG polycistronic transcripts are similar in all three strains. The lower levels of polycistronic transcript compared with gtfG-specific transcript are consistent with previously published results (Sulavik \& Clewell, 1996). (b) rggD-probed RNA of strains $\mathrm{CH} 1, \mathrm{CH} 1$ (pMI226), $\mathrm{CH} 1$ (pVA749) demonstrates the $\sim 1 \mathrm{~kb}$ rggD transcript. There is an increase in the quantity and size of transcripts in the strain carrying plasmid-borne $\operatorname{rgg} D$, which may be due to readthrough in the multicopy pVA749 vector. This has been noted for pAMS57, by Sulavik \& Clewell (1996). (c) dsg-probed RNA of strains $\mathrm{CH} 1, \mathrm{CH} 1$ (pMI226) and $\mathrm{CH} 8961$ shows similar amounts of the $\sim 1.3 \mathrm{~kb} d s g$-specific transcript. (d) ylbN-like-gene-probed RNA of strains $\mathrm{CH} 1, \mathrm{CH}_{1}$ (pMI226) and CH8961 shows similar amounts of a $\sim 2.4 \mathrm{~kb}$ transcript. Blots shown are from mid-exponential-phase cultures grown in FMC medium, and are representative of a minimum of three independent experiments. Similar results were seen when bacteria were grown in Todd-Hewitt broth and when cultures were grown to late-exponential to stationary phase (data not shown). RNA standards were run as indicated, with sizes in kb shown on the right.

(Fig. 4a) indicated that the amount of the $5.4 \mathrm{~kb} g t f G$ specific and $6.4 \mathrm{~kb}$ polycistronic $\mathrm{rgg} / \mathrm{gtfG}$ transcripts were similar in strains $\mathrm{CH} 1$ and $\mathrm{CH} 8942$, suggesting that $d s g$ may be involved in post-transcriptional modifications that result in decreased levels of GTF protein.

\section{Identification of an rgg-like determinant downstream of $g t f G$}

On the same reading strand, $d s g$ is immediately preceded by a DNA inverted repeat and an $867 \mathrm{bp}$ ORF which is $42 \%$ identical to the gtfG positive regulatory deter-

minant, rgg (Fig. 1a). This rgg-like determinant downstream of $g t f G$, designated $\operatorname{rggD}$, encodes a putative $33.6 \mathrm{kDa}$ cytoplasmic protein with a pI of 5.43 . Like Rgg, the encoded RggD has a helix-turn-helix motif (indicated by italics for amino acids 11-64 for Rgg and amino acids 8-61 for RggD in Fig. 5), suggesting a potential DNA-binding function (Wintjens \& Rooman, 1996). In addition to its similarity to $S$. gordonii Rgg, the encoded RggD shares conserved amino acids with characterized Rgg-like proteins from other species (Fig. 5 ), which may provide functional insights into specific domains of these regulatory proteins.

To examine potential functional roles of $r g g D$, strain CH8961, in which $\operatorname{rggD}$ was insertionally inactivated, was constructed. Primer 5'-taGGATCCTATCCACACAGAAACAGAAG-3', with an engineered BamHI site, and primer 5'-AACCGGAGTGCAGATAGA-3', designed to anneal 22 bp upstream of the HindIII site at the $3^{\prime}$ end of pAMS40 (Fig. 1a), were used to amplify an internal fragment of $\operatorname{rgg} D$. The PCR product was digested with restriction enzymes and the $322 \mathrm{bp}$ fragment was cloned into the BamHI and HindIII sites of pVA891 to create pMI8961. Strain CH8961 resulted from the integration of pMI8961 into the strain CH1 chromosome. Despite the similarity of $r g g$ and $r g g D$, there was no cross-reactivity of the internal gene fragments used as probes of $S$. gordonii chromosomal DNA under stringent conditions in Southern blot hybridization analyses (data not shown).

To compare trans effects of $r g g D$ to those of $r g g$ in $S$. gordonii, the $\operatorname{rgg} D$, its putative promoter and its transcriptional termination regions were amplified by PCR using primers 5' $5^{\prime}$ taGATATCCTTCAGCCAAGTCTAGCTTC-3' and 5'-taGATATCCGTAAGGCAAGTTTCATA-3'. The resulting 1397 bp EcoRVflanked fragment was cloned into the compatible HaeIII site of the streptococcal plasmid pVA749. Nucleotide sequence analysis confirmed that the orientation of the rggD fragment in the resulting plasmid, pMI226, was the same as that of $r g g$ in the plasmid pAMS57 previously characterized by Sulavik et al. (1992).

\section{rgg $D$ does not affect GTF activity}

Although their nucleotide and deduced amino acid sequences were similar, the specificities of $\operatorname{rgg}$ and $\operatorname{rggD}$ appeared to differ. Northern blots indicated that disruption of $r g g D$ in strain $\mathrm{CH} 891$ did not affect levels of $\mathrm{rgg} / \mathrm{gtfG}$ or $g t f G$ transcript (Fig. 4a). The GTF activities of strains CH1 and CH8961 were similar (Fig. 3a), indicating that disruption of $\mathrm{rggD}$ did not affect potential post-transcriptional modifications that would result in altered levels of GTF activity. Effects of plasmid-borne $r g g$ and $r g g D$ were also specific. Plasmidborne rgg in pAMS57 has been shown to increase GTF activity approximately sixfold in the parental strain CH1; pAMS57 has also been shown to increase GTF activity to the same level in strain DS512 (Sulavik et al., 1992). Due to a frameshift mutation resulting in a premature translation stop in $\mathrm{rgg}$, strain DS512 has only 


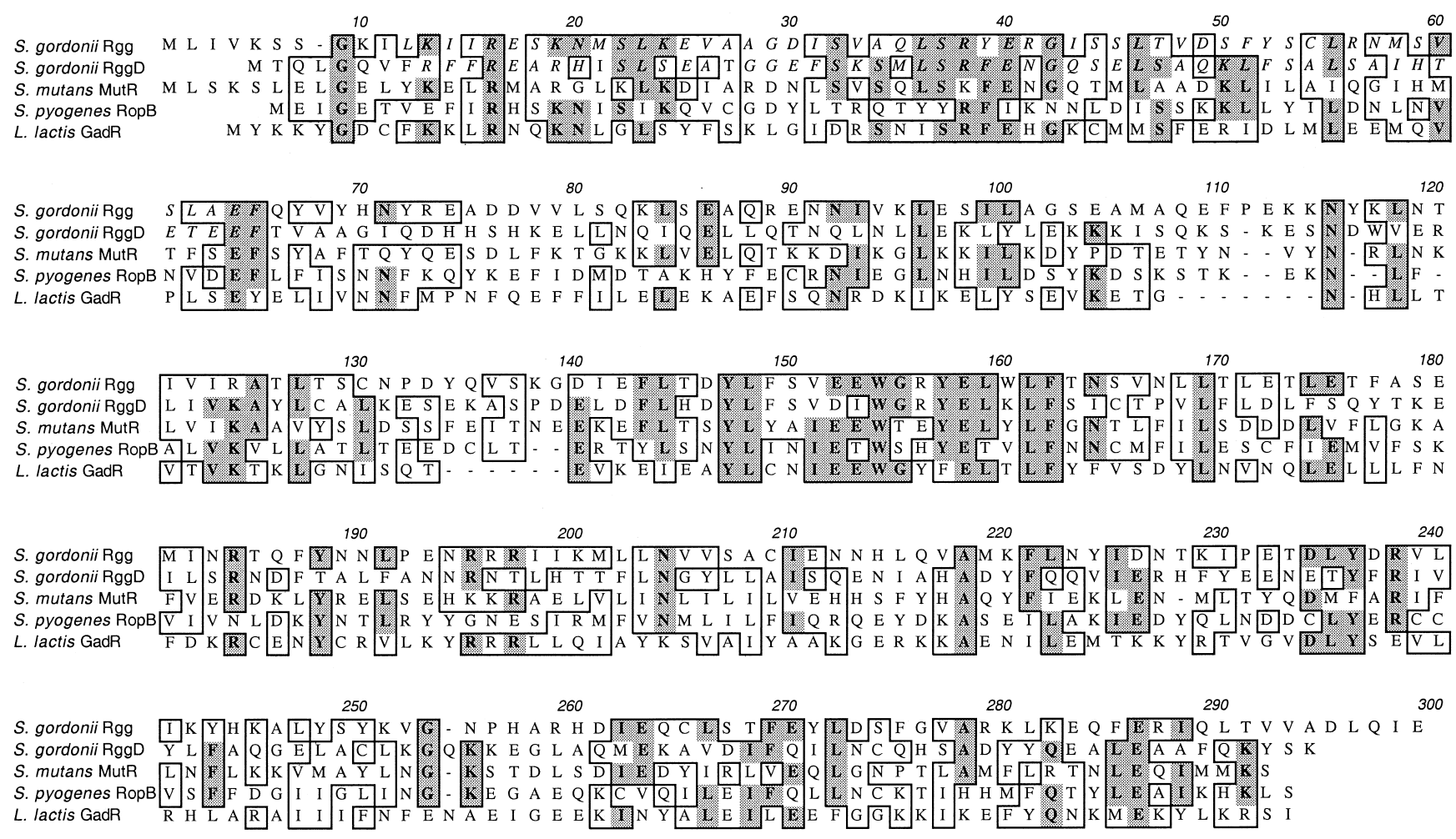

Fig. 5. Comparison of deduced amino acid sequences of S. gordonii Rgg and RggD to other characterized Rgg-like proteins encoded by S. mutans mutR (Qi et al., 1999), S. pyogenes ropB (Lyon et al., 1998) and L. lactis gadR (Sanders et al., 1998) using CLUSTAL analysis (Thompson et al., 1994). Deduced amino acids of S. gordonii Rgg and RggD in the putative helix-turn-helix motif (Bateman et al., 2000), which may function in DNA binding (Wintjens \& Rooman, 1996), are shown in italics. Conserved regions of the similar proteins are shown in shaded blocks.

$\sim 3 \%$ of the parental level of GTF activity (Sulavik \& Clewell, 1996). However, plasmid-borne $\operatorname{rggD}$ did not increase GTF activity in strains CH1 or DS512 to a level above that of the plasmid-free strain (Fig. 3b). Thus, $\operatorname{rgg} D$ in trans did not affect GTF activity, even in the absence of a functional chromosomal rgg. These results suggest that $\operatorname{rggD}$ does not influence gtfG expression and may provide important insights into the specificity of $r g g$-like genes.

\section{Effects of rggD on transcription of flanking genes}

To determine if $r g g D$ and $d s g$ were co-transcribed, strain CH1 RNA was probed with internal fragments of $r g g D$ and $d s g$ in Northern blots (Fig. $4 \mathrm{~b}, \mathrm{c}$ ). A $\sim 1.0 \mathrm{~kb}$ rggD-specific transcript and a $\sim 1.3 \mathrm{~kb} d$ sg-specific transcript were detected. No polycistronic transcript was noted.

Previously characterized $r g g$-like genes positively regulate transcription of adjacent genes (Sulavik \& Clewell, 1996; Lyon et al., 1998; Sanders et al., 1998; Qi et al., 1999; Chaussee et al., 1999). Furthermore, rgg-like genes are often separated from the gene they regulate by DNA secondary structures, such as inverted repeats. Accordingly, the inverted repeats between $\operatorname{rggD}$ and $d s g$ suggested the possibility that $\operatorname{rgg} D$ might affect $d s g$ transcription. However, in Streptococcus pyogenes, the rgg-like gene that regulates the gene for streptococcal erythrogenic toxin $\mathrm{B}$, speB, is transcribed in the divergent reading direction (Lyon et al., 1998) Therefore, the possibility that $\operatorname{rgg} D$ regulates the gene further downstream was also investigated. Nucleotide sequencing identified a $531 \mathrm{bp}$ ORF downstream of $\operatorname{rggD}$, in the divergent reading direction (Fig. 1a). This gene encodes a predicted $19 \cdot 9 \mathrm{kDa}$ cytoplasmic protein with a pI of 3.89 . Examination of genome databases indicated that this gene is most similar to the $y l b N$-like gene of unknown function of Lactococcus lactis subsp. cremoris. The $S$. gordonii ylbN-like gene is associated with $\mathrm{a} \sim 2.4 \mathrm{~kb}$ transcript (Fig. $4 \mathrm{~d}$ ), suggesting that it is co-transcribed with determinants further downstream. Indeed, partial sequence of an ORF, designated orf8 in Fig. 1(a), showed a putative ribosome-binding site for translation of the encoded protein, but no apparent transcriptional termination sequences were noted in the region between the $y l b N$-like gene and orf8.

Northern blot analyses were used to examine the transcription of $d s g$ and $y l b N$-like genes in strain CH8961 (in which $\operatorname{rgg} D$ was insertionally inactivated) and in a parental strain carrying additional copies of $r g g D$ in pMI226 (Fig. 4b, c). Unexpectedly, the results did not indicate any differences in transcription of either $d s g$ or $y l b N$ in these strains compared with the parental strain CH1. Recent studies in S. pyogenes have indicated 
that an $r g g$-like determinant influences transcription in stationary-phase cells (Chaussee et al., 2001); hence the $S$. gordonii strains were also examined in lateexponential to stationary growth phase. However, as seen in the mid-exponential-phase cells, no differences were evident (data not shown). Similar results were also seen when the $S$. gordonii strains were grown in defined FMC medium and in complex Todd-Hewitt broth, indicating that components of these different media did not influence potential regulation of either gene by $\operatorname{rgg} D$. These results do not preclude the possibility that $\operatorname{rgg} D$ affects transcription of the $d s g$ or $y l b N$-like genes under conditions other than those in the present study.

\section{Comparison of streptococcal chromosomal regions}

The finding that $r g g D$ did not influence transcription of the adjacent genes raised the possibility that $\operatorname{rgg} D$ regulates distally located genes on the $S$. gordonii chromosome. This hypothesis is supported by the comparison of similar chromosomal regions using available genome data for other streptococcal species (Fig. 1b). S. pyogenes has $h t p X-$ and $y l b N$-like genes adjacent to each other. However, S. gordonii has four genes between the $h t p X$ and $y l b N$-like genes, i.e. two convergent genes, $g t f G$ and $d s g$, which are each preceded by similar rgglike genes. Both Streptococcus mutans and Streptococcus pneumoniae have hypothetical ATP-binding proteins with no $r g g$-like determinants on the opposite reading strand between their $h t p X-$ and $y l b N$-like genes. These hypothetical genes, encoding ATP-binding proteins, do not have significant similarities to the $S$. gordonii rgg-flanked gtfG and $d s g$ genes. However, there is a $d s g$-like gene $(37 \%$ identity at the nucleotide level; $57 \%$ similarity of the encoded putative proteins) distally located on the $S$. pneumoniae chromosome between putative histidine tRNA ligase and dihydroxyacid dehydratase genes. These findings suggest that the $S$. gordonii determinants between the $h t p X$ and $y l b N-$ like genes may have resulted from recombinations between similar DNA regions within the $S$. gordonii chromosome, or via horizontal gene transfer. It is possible that such a recombination or rearrangement resulted in distancing $\mathrm{rggD}$ from the $S$. gordonii gene which it regulates.

\section{DISCUSSION}

Examination of the region downstream of $g t f G$ led to the identification of a gene, designated $d s g$, which affects the level of GTF activity. The similar amounts of $g t f G$ seen in Northern blots, together with the sequence and structural similarities of the encoded Dsg to a protein involved in polysaccharide biosynthesis in Gram-negative organisms (Skurnik et al., 1995), support the possibility that $d s g$ may be involved in some posttranscriptional and/or post-translational modifications that affect the level of GTF. Insertional inactivation of $d s g$ resulted in a decrease of approximately $40 \%$ in GTF activity. Although colonies of these $d s g$ mutants were hard and cohesive $\mathrm{Spp}^{+}$on sucrose agar plates, they also had a glassy, more translucent appearance than parental colonies. It is not known if this phenotypic change is due to the approximately $60 \%$ level of GTF activity, or due to changes in the glucan polymers themselves. Such changes in synthesized glucans could potentially result from conformational changes of the active enzyme when it is near or attached to the cell surface (Kopec et al., 1997), as might occur if there were differences in the extracellular transport of GTF. Mutations in S. gordonii gtf $G$ can lead to changes in the proportions of $\alpha 1,3-$ and $\alpha 1,6$-glucosidic linkages in the synthesized glucans, and result in changes in the detectable levels of GTF activity: as measured by both $\left[\right.$ glucose $\left.-{ }^{14} \mathrm{C}\right]$ sucrose incorporation into glucan and GTF activity gel assays (Vickerman et al., 1996). Analysis of the glucans produced by colonies of strain CH8942 grown on sucrose agar plates was not done in the present study. Spp ${ }^{-}$strains, which produce soft colonies on sucrose agar plates, have been reported to have less than $30 \%$ the parental level of GTF activity (Tardif et al., 1989). Changes in Spp have also been associated with changes in the amino acid composition of the GTF enzyme (Vickerman et al., 1996). Development of the Spp ${ }^{-}$and changes in GTF activity have been associated with the ability of $S$. gordonii cells to be released from biofilm surfaces (Vickerman et al., 1991), which may provide important ecological advantages for cells growing in dental plaque. Thus, genes such as rgg, $g t f G$ and $d s g$, involved in varying the quantity or quality of glucans produced by the GTF enzyme of the commensal S. gordonii, may play important roles in oral microbial ecology.

Directly $5^{\prime}$ to $d s g$, an $r g g$-like determinant, $r g g D$, was identified: this is the third $r g g$-like determinant reported in S. gordonii. In addition to the originally identified positive $\operatorname{ttfG}$ regulator, $r g g$ (Sulavik et al., 1992), an rgglike determinant, iviB, was recovered from a rabbit endocarditis model using in vivo expression technology (Kilic et al., 1999); this suggests that $i v i B$, or possibly a gene(s) that it regulates, is required for survival in vivo. Multiple rgg-like genes are present in $S$. pyogenes (Ferretti et al., 2001), S. mutans (http:// www.genome.ou.edu) and S. pneumoniae (http:// www.tigr.org), suggesting that rgg-like genes make up a relatively widely occurring family of streptococcal regulatory genes. Under the conditions in the present studies, the $S$. gordonii gene that $r g g D$ regulates was not identified. Based upon the findings that most rgg-like determinants regulate adjacent genes (Sulavik \& Clewell, 1996; Lyon et al., 1998; Sanders et al., 1998; Qi et al., 1999; Chaussee et al., 1999) it was hypothesized that $\operatorname{rggD}$ would regulate the S. gordonii dsg or $y l b N$ like genes. Northern blots did not confirm this hypothesis. However, it is possible that changes in the levels of the $d s g$ and $y l b N$ transcript in strains with disruption or additional copies of $\mathrm{rggD}$ were too subtle to be detected by Northern blot hybridization analyses. Nevertheless, the dramatic sixfold differences in gtfG transcription due to $\mathrm{rgg}$ (Sulavik \& Clewell, 1996), along with the effects of $\operatorname{rgg}(\operatorname{rop} B)$ on transcription of speB (Lyon et al., 1998), were readily apparent on Northern blots, suggesting that the magnitude of posi- 
tive regulation by $r g g$-like genes should be clearly evident by this assay. It is possible that $\operatorname{rggD}$ regulates either of the adjacent genes under conditions other than those in these studies. Although transcripts were examined from mid-exponential through to stationary growth phase in defined as well as complex media, there are innumerable conditions that could affect genetic regulation. Comparison of the chromosomal organizations of related streptococci, which show that the genes present in the regions between $h t p X$ - and $y l b N$-like genes differ among these species, suggest that DNA recombinations and rearrangements may have occurred in these intergenic regions. This finding gives support to additional speculation that $r g g D$ may regulate distally located $S$. gordonii gene(s) from which it was distanced by recombinational events. Additional S. gordonii genome sequence data may provide insights into this possibility.

\section{ACKNOWLEDGEMENTS}

This work was supported by Public Health Service grant DE11090 from the National Institutes of Health.

\section{REFERENCES}

Altschul, S. G., Gish, W., Miller, W., Myers, E. W. \& Lipman, D. J. (1990). Basic local alignment search tool. J Mol Biol 215, 403-410.

Ausubel, F. M., Brent, B., Kingston, R. E., Moore, D. D., Seidman, J. G., Smith, J. A. \& Struhl, K. (1987). Current Protocols in Molecular Biology. New York: Wiley.

Bateman, A., Birney, E., Durbin, R., Eddy, S. R., Howe, K. L. \& Sonnhammer, E. L. (2000). The pfam protein families database. Nucleic Acids Res 28, 263-266.

Chaussee, M. S., Ajdic, D. \& Ferretti, J. J. (1999). The rgg gene of Streptococcus pyogenes NZ131 positively influences extracellular SPE B production. Infect Immun 67, 1715-1722.

Chaussee, M. S., Watson, R. O., Smoot, J. C. \& Musser, J. M. (2001). Identification of Rgg-regulated exoproteins of Streptococcus pyogenes. Infect Immun 69, 822-831.

Ferretti, J. J., McShan, W. M., Ajdic, D. \& 20 other authors (2001). Complete genome sequence of an M1 strain of Streptococcus pyogenes. Proc Natl Acad Sci US A 98, 4658-4663.

Frandsen, E. V. G., Pedrazzoli, V. \& Kilian, M. (1991). Ecology of viridans streptococci in the oral cavity and pharynx. Oral Microbiol Immunol 6, 129-133.

Fujiwara, T., Hoshino, T., Ooshima, T., Sobue, S. \& Hamada, S. (2000). Purification, characterization, and molecular analysis of the gene encoding glucosyltransferase from Streptococcus oralis. Infect Immun 68, 2475-2483.

Grahame, D. A. \& Mayer, R. M. (1984). The origin and composition of multiple forms of dextransucrase from Streptococcus sanguis. Biochim Biophys Acta 786, 42-48.

Haisman, R. J. \& Jenkinson, H. F. (1991). Mutants of Streptococcus gordonii Challis overproducing glucosyltransferase. J Gen Microbiol 137, 483-489.

Hofmann, K. \& Stoffel, W. (1993). TMbase-a database of membrane spanning proteins segments. Biol Chem Hoppe-Seyler 374, 166.

Kilic, A. O., Herzberg, M. C., Meyer, M. W., Zhao, X. \& Tao, L. (1999). Streptococcal reporter gene-fusion vector for identification of in vivo expressed genes. Plasmid 42, 67-72.

Kopec, L. K., Vacca-Smith, A. M. \& Bowen, W. H. (1997). Struc- tural aspects of glucans formed in solution and on the surface of hydroxyapatite. Glycobiology 7, 929-934.

Lawson, J. \& Gooder, H. (1970). Growth and development of competence in the group H streptococci. J Bacteriol 102, 820-825. Lyon, W. R., Gibson, C. M. \& Caparon, M. G. (1998). A role for trigger factor and an rgg-like regulator in the transcription, secretion and processing of the cysteine proteinase of Streptococcus pyogenes. EMBO 17, 6263-6275.

Macrina, F. L., Tobian, J. A., Jones, K. R. \& Evans, R. P. (1981). Molecular cloning in the streptococci. In Genetic Engineering of Microorganisms for Chemicals, pp. 195-210. Edited by A. Hollaender, R. DeMoss, S. Kaplan, J. Konisky, D. Savage \& R. Wolfe. New York: Plenum.

Macrina, F. L., Evans, R. P., Tobian, J. A., Hartley, D. L., Clewell, D. B. \& Jones, K. R. (1983). Novel shuttle plasmid vehicles for Escherichia-Streptococcus transgeneric cloning. Gene 25, 145-150.

Nielsen, H., Engelbrecht, J., Brunak, S. \& von Heijne, G. (1997). Identification of prokaryotic and eukaryotic signal peptides and prediction of their cleavage sites. Protein Eng 10, 1-6.

Qi, G., Chen, P. \& Caufield, P. W. (1999). Functional analysis of the promoters in the lantibiotic mutacin II biosynthetic locus in Streptococcus mutans. Appl Environ Microbiol 65, 652-658.

Reeves, P. R., Hobbs, M., Valvano, M. A. and 8 other authors (1996). Bacterial polysaccharide synthesis and gene nomenclature. Trends Microbiol 4, 495-503.

Sanders, J. W., Leenhouts, K., Burghoorn, J., Brands, J. R., Venema, G. \& Kok, J. (1998). A chloride-inducible acid resistance mechanism in Lactococcus lactis and its regulation. Mol Microbiol 27, 299-310.

Schnaitman, C. A. \& Klena, J. D. (1993). Genetics of lipopolysaccharide biosynthesis in enteric bacteria. Microbiol Rev 57, 655-682.

Skurnik, M., Venho, R., Toivanen, P. \& Al-Hendy, A. (1995). A novel locus of Yersinia enterocolitica serotype $\mathrm{O}: 3$ involved in lipopolysaccharide outer core biosynthesis. Mol Microbiol 17, 575-594.

Sulavik, M. C. \& Clewell, D. B. (1996). Rgg is a positive transcriptional regulator of the Streptococcus gordonii gtfG gene. J Bacteriol 178, 5826-5830.

Sulavik, M. C., Tardif, G. \& Clewell, D. B. (1992). Identification of a gene, $r g g$, which regulates expression of glucosyltransferase and influences the Spp phenotype of Streptococcus gordonii Challis. J Bacteriol 174, 3577-3586.

Tardif, G., Sulavik, M., Jones, G. W. \& Clewell, D. B. (1989). Spontaneous switching of the sucrose-promoted colony phenotype in Streptococcus sanguis. Infect Immun 57, 3945-3948.

Terleckyj, B., Willett, N. P. \& Shockman, G. D. (1975). Growth of several cariogenic strains of oral streptococci in a chemically defined medium. Infect Immun 11, 649-655.

Thompson, J. D., Higgins, D. G. \& Gibson, T. J. (1994). CLUSTAL W : improving the sensitivity of progressive multiple sequence alignment through sequence weighting, position-specific gap penalties and weight matrix choice. Nucleic Acids Res 22, 4673-4680.

Vickerman, M. M. \& Clewell, D. B. (1997). Deletions in the carboxyl terminal region of Streptococcus gordonii glucosyltransferase affect cell-associated enzyme activity and sucroseassociated accumulation of growing cells. Appl Environ Microbiol 63, 1667-1673.

Vickerman, M. M., Clewell, D. B. \& Jones, G. W. (1991). Ecological implications of glucosyltransferase phase variation in Streptococcus gordonii. Appl Environ Microbiol 57, 3648-3651. 
Vickerman, M. M., Sulavik, M. C. \& Clewell, D. B. (1995). Oral streptococci with genetic determinants similar to the glucosyltransferase regulatory gene, rgg. Infect Immun 63, 4524-4527.

Vickerman, M. M., Sulavik, M. C., Minick, P. E. \& Clewell, D. B. (1996). Changes in the carboxyl terminal repeat region affect extracellular activity and glucan products of Streptococcus gordonii glucosyltransferase. Infect Immun 64, 5117-5128.

Vickerman, M. M., Jones, G. W. \& Clewell, D. B. (1997a). Molecular analysis of representative Streptococcus gordonii Spp phase variants reveals no differences in the glucosyltransferase structural gene, gtfG. Oral Microbiol Immunol 12, 82-90.

Vickerman, M. M., Sulavik, M. C., Nowak, J. D., Gardner, N. M.,
Jones, G. W. \& Clewell, D. B. (1997b). Nucleotide sequence analysis of the Streptococcus gordonii glucosyltransferase gene, gtfG. DNA Seq 7, 83-95.

Vickerman, M. M., Mather, N. M., Minick, P. E. \& Edwards, C. A. (2002). Initial characterization of the Streptococcus gordonii htpX gene. Oral Microbiol Immun (in press).

Wintjens, R. \& Rooman, M. (1996). Structural classification of HTH DNA-binding domains and protein-DNA interaction modes. J Mol Biol 262, 294-313.

Received 6 June 2001; revised 6 July 2001; accepted 24 July 2001. 